\title{
Bronchial needle aspiration in the diagnosis of bronchial carcinoma
}

\author{
G BUIRSKI, PMA CALVERLEY, NJ DOUGlAS, D LAMB, M McINTYRE, \\ MF SUDLOW, H WHITE
}

From the City Hospital, Edinburgh

\begin{abstract}
Sixty consecutive patients with central bronchial carcinomas were studied by fibreoptic bronchoscopy. In all forceps biopsy and bronchial needle aspiration were performed, and in 54 bronchial brushings were obtained. The combination of bronchial brushings and forceps biopsy diagnosed bronchial carcinoma of a defined cell type in $80 \%$ of patients. Bronhial needle aspiration was the most effective single technique giving a cytological diagnosis in $80 \%$ of patients, and when all three techniques were combined the positive rate increased to $92 \%$. No major complications occurred using bronchial needle aspiration. Needle aspiration was particularly helpful when sampling from lesions in the upper lobes where forceps biopsies were technically difficult, from tumours lying submucosally, and from abnormalities caused by extrinsic compression. We conclude that bronchial needle aspiration should be used routinely, together with other sampling techniques, in the diagnosis of central bronchial carcinoma with the fibreoptic bronchoscope.
\end{abstract}

\begin{abstract}
Although many uses have been found for the fibreoptic bronchoscope, its primary role still remains in the diagnosis of bronchial carcinoma. Three major sampling techniques are used with the fibreoptic bronchoscope-bronchial brushings, forceps biopsy, and bronchial washings.

Needle aspiration is an effective means of obtaining cytological specimens but until recently its only roles in the diagnosis of primary pulmonary tumours were for percutaneous transthoracic needle biopsy ${ }^{1}$ and to aspirate paratracheal tumours with the rigid bronchoscope. ${ }^{2}$

A technique for needle aspiration biopsy through the fibreoptic bronchoscope has now been reported by Oho and his colleagues from Japan. ${ }^{3}$ We have been using a similar technique and report a comparison of needle aspiration biopsy with other sampling procedures.
\end{abstract}

\section{Methods}

Sixty consecutive patients who had lesions with the bronchoscopic appearance of carcinoma were studied, using either an Olympus BF-B2 or BF-B3 bronchoscope which was passed transnasally. Three

Address for reprint requests: Dr MF Sudlow, City Hospital, Greenbank Drive, Edinburgh EH10 5SB. different sampling techniques were employed to obtain pathological material. First, bronchial brushings were performed and the material obtained smeared on to glass slides and rapidly fixed in alcohol. Bronchial needle aspiration was performed next using the Olympus NM3K injection needle originally designed for sclerosing oesophageal varices with a fibreoptic gastroscope. ${ }^{4}$ The aspiration needle was introduced through the bronchoscope and a $4 \mathrm{~mm}$ needle advanced under direct vision into the lesion. With the needle in situ negative pressure was exerted using a $20 \mathrm{ml}$ disposable syringe held for approximately 10 seconds, after which the plunger was slowly released. The needle was then withdrawn into its sheath, removed from the bronchoscope and the aspirate smeared on to glass slides which were then fixed in alcohol. Needle aspiration was repeated until two adequate slide specimens had been obtained. Immediately after each aspiration, the system was flushed through with saline followed by air to prevent any blockage or carry over of cellular material between aspiration biopsies. Between cases the needle was soaked in Cidex for 10 minutes, to eliminate the risk of hepatitis $B$ transfer between patients. This sterilisation procedure was repeated for a period of 12 hours at the end of each bronchoscopy session.

After aspiration at least two good forceps biopsies 
were obtained using the Olympus FB14C forceps. Bronchial washings were not performed routinely as they have been shown to be of no additional value in the diagnosis of central lesions. 5 After bronchoscopy, bronchial biopsies and brushings were reviewed by two pathologists. The needle aspiration specimens were sent to a different cytopathologist for review with no further histological evidence available. On completion of the study the bronchial brushings were reassessed by the same cytopathologist. Our criterion for the diagnosis of bronchial carcinoma on cytology was the ability to state the cell type from which the tumour originated. All interpretations other than bronchial carcinoma of a specific cell type were regarded as negative. On no occasion were malignant cells found to which a cell type could not be attributed.

\section{Results}

All 60 patients studied had bronchial forceps biopsy and needle aspiration biopsy performed and in 54 patients bronchial brushings were obtained in addition.

In 54 of the 60 patients with clinical evidence of bronchial carcinoma the diagnosis was eventually confirmed by either samples obtained at bronchoscopy or by sputum cytology, lymph node biopsy, or thoracotomy. In each of these cases where the diagnosis was made by only one cytological technique, this was subsequently confirmed by one or more further sampling methods. Of the remaining six patients, three had only needle aspiration biopsies diagnostic of bronchial carcinoma, and in three patients the diagnosis remained unproven, though the clinical and radiological features were strongly suggestive.

When diagnostic techniques were compared (table 1) bronchial brushings and bronchial biopsy had diagnostic rates of $65 \%$ and $67 \%$ respectively. Needle aspiration biopsy alone had a positive diagnostic rate of $80 \%$, although this difference was not statistically significant using the chi-squared test. The combination of bronchial biopsy and brushings provided a positive diagnosis also in $80 \%$ of patients. When all three sampling methods were combined, the diagnostic rate increased to $92 \%$ (figure). The combination of forceps biopsy and

Table 1 Comparison of diagnostic techniques used

\begin{tabular}{llll}
\hline Technique & Positive & Negative & Total \\
\hline Brushings & $35(65 \%)$ & 19 & 54 \\
Biopsy & $40(67 \%)$ & 20 & 60 \\
Needle aspiration & $48(80 \%)$ & 12 & 60 \\
\hline
\end{tabular}

needle aspiration biopsy gave a positive diagnosis in all but one patient found to have bronchial carcinoma using the three combined sampling techniques.

We found that there was no discrepancy in cytological cell types between the techniques when using the World Health Organisation classification. Two needle aspiration biopsies contained atypical cells which were not diagnostic of bronchial carcinoma, and in one the diagnosis was subsequently confirmed by other means. Bronchial

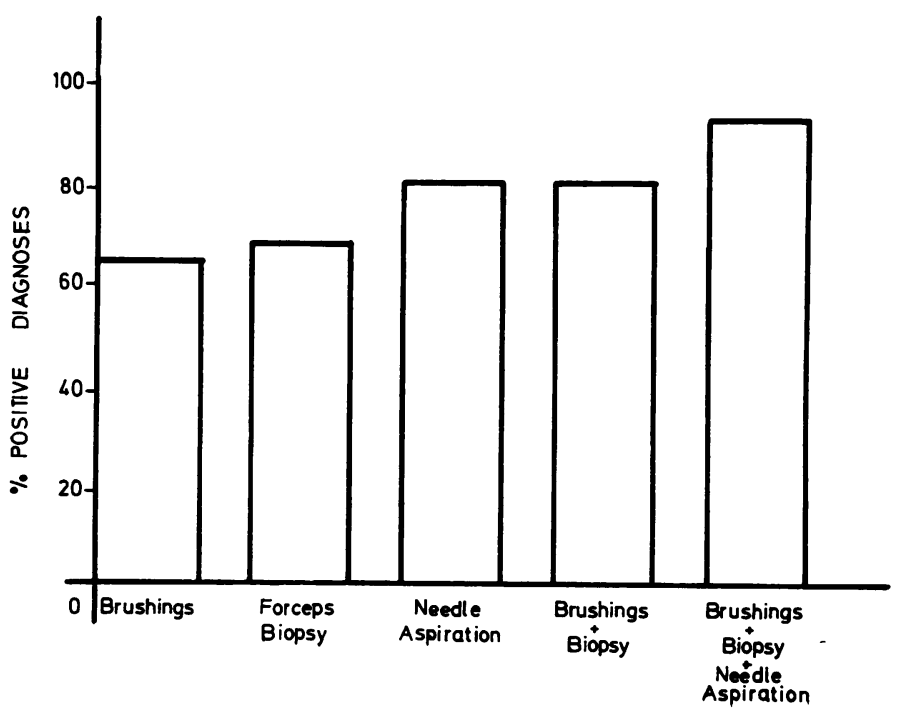

Figure Histogram comparing combined effects of different sampling techniques. 
brushings yielded atypical cells in two patients, both of whom were subsequently proved by other methods to have bronchial carcinoma. The histological cell types found in our series are summarised in table 2.

Table 2 Histological distribution of bronchial carcinoma in the 60 patients studied

\begin{tabular}{lclr}
\hline Histology & Male & Female & Total \\
\hline Squamous cell & 32 & 9 & $41(68 \%)$ \\
Small cell & 9 & 6 & $15(25 \%)$ \\
Carcinoid & 1 & - & $1(2 \%)$ \\
Unknown & 2 & 1 & $3(5 \%)$ \\
\hline
\end{tabular}

\section{Discussion}

There have been many studies comparing the diagnostic value of bronchial brushings and forceps biopsy in the diagnosis of central bronchial carcinoma, ${ }^{5-7}$ but few have used the confident diagnosis of a tumour of a definite cell type as a basis for comparison of techniques. Using this criterion for bronchial brushings, Kvale et $a l^{5}$ obtained a $77 \%$ positive diagnostic rate, and Solomon et al ${ }^{6}$ diagnosed only six of 11 patients with central lesions. Zavala $^{7}$ and Funahashi et $a^{8}$ have achieved much higher positive bronchial brushing results of $93 \%$ and $83 \%$ respectively, though both used a different method of obtaining material. In these studies the bronchoscope was introduced via an endotracheal tube, and once brushings had been obtained both the bronchoscope and brush were removed from the patient and the tips of both instruments agitated in Ringer's solution. Although this difference in technique may be of importance, Parker et al ${ }^{9}$ has shown no significant increase in the diagnostic yield between the withdrawn and non-withdrawn bronchial brushing procedures. Thus, our rate of $65 \%$ for bronchial brushings is similar to that of others using the same cytological criteria and method of sampling.

The accuracy of bronchial biopsy in the diagnosis of bronchial cacinoma varied in different studies from $71 \%$ to $97 \% 57810$ whereas we achieved an accuracy of $67 \%$. Twenty-eight of our patients had lesions in the upper lobe, 17 found proximally at the lobar entrance, while the remainder were distal, either in segmental bronchi or beyond the lingular orifice (table 3). In 14 of these 17 proximal lesions, forceps biopsy and needle aspiration each gave positive diagnoses, while in the 11 distal lesions forceps biopsy was only positive in four compared to 10 positive needle aspiration biopsies. Technical difficulties contributed to the negative biopsy results in
Table 3 Numbers of positive diagnoses by sampling methods at varying tumour sites. Figures in parentheses denote number of samples obtained when less than total

\begin{tabular}{|c|c|c|c|c|c|}
\hline Sites & & $\begin{array}{l}\text { Total number } \\
\text { of lesions } \\
\text { site }\end{array}$ & $\begin{array}{l}\text { Needle } \\
\text { aspiration }\end{array}$ & Biopsy & Brushings \\
\hline \multicolumn{2}{|c|}{ Major bronchi* } & 19 & 14 & 14 & $12(17)$ \\
\hline \multirow{2}{*}{ Upper lobes } & Proximal & 117 & 14 & 14 & $10(16)$ \\
\hline & Distal & 11 & 10 & 4 & $6(9)$ \\
\hline \multirow{2}{*}{ Lower lobes } & Proximal & 5 & 3 & 3 & 2 \\
\hline & Distal & 8 & 7 & 5 & $5 \quad(7)$ \\
\hline
\end{tabular}

*Left and right main bronchi and bronchus intermedius.

these latter cases. When the forceps were introduced, flexion of the bronchoscope tip was reduced, thus impairing accurate biopsy from the lesion, if indeed the lesion could be reached at all. Aspiration biopsy did not limit flexion of the bronchoscope and thus all visible lesions could be aspirated without difficulty. There were no differences in the diagnostic yield between forceps biopsy and needle aspiration biopsy in other sites.

In a further three patients, lesions were identified in the bronchus intermedius, and had the appearance of submucosal infiltration. Forceps biopsies were attempted, but because of the flat angle of incidence the forceps could not grip the bronchial wall sufficiently to obtain a deep specimen. At transbronchial aspiration the needle was seen to penetrate the bronchial wall and good samples of pathological material were obtained. Similarly, in four patients where the abnormality was the result of extrinsic compression, transbronchial aspiration was positive in all and forceps biopsy in three. This ability to obtain samples from outwith the bronchial wall confirms a suggestion by Oho and his colleagues. ${ }^{3}$

No major complications occurred using bronchial needle aspiration in this study. There was rarely much haemorrhage after aspiration biopsy, thus enabling the operator to take multiple samples without obscuring vision. In many patients, the site of $N$ aspiration biopsy could be identified by pinpoint $N$ haemorrhage, allowing the operator to adjust his $\mathrm{N}$ target area for subsequent aspirations. Bronchial $\omega$ needle aspiration provides a simple means of improving the diagnostic yield from sites technically inaccessible to forceps biopsy, and may also prove $\underset{\mathscr{D}}{\mathscr{D}}$ to be valuable in the diagnosis of peripheral lesions ? under fluoroscopic screening or in transcarinal aspirations as part of a staging procedure in this $\underset{\mathbb{D}}{\vec{D}}$ disease.

We would like to acknowledge the financial help given by the Melville Trust for the purpose of $\delta$ 
buying equipment, and to Professor DC Flenley, Dr NW Horne, Dr GJR McHardy, and Dr A Seaton for allowing us to study patients under their care.

\section{References}

${ }^{1}$ Grech P, Gray RB, Lambourne CA, Clark RA, Townshend RH. Aspiration needle biopsy for solitary peripheral lung lesions: a review of 50 examinations. $\mathrm{Br} J \mathrm{Dis}$ Chest 1978;72:235-41.

${ }^{2}$ Wang KP, Terry P, March B. Bronchoscopic needle aspiration biopsy of paratracheal tumors. Am Rev Respir Dis 1978;118:17-21.

${ }^{3}$ Oho K, Kato H, Ogawa I, Hayashi N, Hayata Y. A new needle for trans-fibreoptic bronchoscopic use. Chest $1979 ; 76: 492$.

4 Williams KGD, Dawson JL. Fibreoptic injection of oesophageal varices. $\mathrm{Br}$ Med J 1979;2:766-7.
${ }^{5}$ Kvale PA, Bode FR, Kini S. Diagnostic accuracy in lung cancer: comparison of techniques used in fibreoptic bronchoscopy. Chest 1976;69:752-9.

- Solomon DA, Solliday NH, Gracey DR. Cytology in fibreoptic bronchoscopy: comparison of bronchial brushings, washing and post-bronchoscopy sputum. Chest 1974;65:616-9.

7 Zavala DC. Diagnostic fibreoptic bronchoscopy: techniques and results of biopsy in 600 patients. Chest 1975; 68:12-9.

${ }^{8}$ Funahashi A, Browne TK, Houser WC, Hranicka LJ. Diagnostic value of bronchial aspirate and postbronchoscopic sputum in fibreoptic bronchoscopy. Chest 1979;76:514-7.

- Parker RL, Haesaert SP, Kovnat DM, Bachus B, Snider GL. Bronchial brushings in bronchogenic carcinoma: factors influencing cellular yield and diagnostic accuracy. Chest 1977;71:341-5.

10 Webb J, Clarke SW. A comparison of biopsy results using rigid and fibreoptic bronchoscopes. $\mathrm{Br} J$ Dis Chest 1980; 74:81-3. 